RAIRO Operations Research

RAIRO Oper. Res. 37 (2003) 311-323

DOI: $10.1051 /$ ro: 2004004

\title{
CONSISTENCY CHECKING WITHIN LOCAL SEARCH APPLIED TO THE FREQUENCY ASSIGNMENT WITH POLARIZATION PROBLEM
}

\author{
Michel Vasquez $^{1}$, Audrey Dupont ${ }^{1}$ and Djamal Habet ${ }^{1}$
}

\begin{abstract}
We present a hybrid approach for the Frequency Assignment Problem with Polarization. This problem, viewed as Max-CSP, is treated as a sequence of decision problems, CSP like. The proposed approach combines the Arc-Consistency techniques with a performed Tabu Search heuristic. The resulting algorithm gives some high quality solutions and has proved its robustness on instances with approximately a thousand variables and nearly ten thousand constraints.
\end{abstract}

Keywords. Filtering techniques, consistency checking, Tabu Search.

\section{INTRODUCTION}

The ever-increasing demand for communication, coupled with the limited number available spectra, have made frequency assignment more and more difficult to accomplish effectively. Optimization of this process has therefore become a major issue for network administration and deployment, both civil and military. The Frequency Assignment Problem with Polarization $(F A P P)$ can be formalized as a Max-CSP which is known to be NP-hard.

Because the data problem to be optimized was very large, we decided to adopt a local search method. From among all the existing algorithms, we chose Tabu Search, introduced by Glover in [8]. The specific structure of the FAPP constraint network led us to apply local filtering techniques. Consequently, an arc-consistency procedure, AC, was embedded in the Tabu Search framework to reduce the search

\footnotetext{
1 Centre LGI2P, École des mines d'Alès, site EERIE, 30035 Nîmes Cedex 1, France;

e-mail: vasquez, dupont, habet@site-eerie.ema.fr
}

(C) EDP Sciences 2004 
space. Hence, the consistency checking concept occurred twice (during the resolution): firstly in the filtering pre-processing, and secondly in the kernel of the neighborhood design.

After presenting the physical and formal definitions of the FAPP problem in Section 2, we describe in Section 3 our general approach to solve it. Finally, a large number of experimental results are discussed and compared in Section 4, in order to highlight the advantages obtained by combining a constraint programming tool with the local search heuristic.

\section{Problem Definition}

\subsection{Physical Description}

The FAPP consists in finding an optimal frequency allocation in hertzian telecommunication networks. The network is composed of a set of sites in which the transmission devices (antennae connected to emitters or receptors) are located. A hertzian connection joins two geographic sites by one or more paths. A path is a uni-directional radio-electric bond, established between antennae at distinct sites, which has a given frequency and polarization.

A frequency resource is therefore a (frequency, polarization) pair in which the components are respectively associated to the carrying frequency of the transmitted signal, and the wave polarization. The polarization is simply either positive or negative. Accordingly, we define the path domain as a set of available resources. This set contains the frequency domain $F_{i}$, and the polarization information $P_{i}$, which may include a required polarization.

A frequency allocation consists in assigning a $\left(f_{i}, p_{i}\right)$ pair for each path $i$ which satisfies certain radio-electric compatibility constraints (1, 2, and 3), and minimal distance constraints to avoid interference (4):

1. frequencies equality or not across paths $i$ and $j: f_{i}=f_{j}$ or $f_{i} \neq f_{j}$;

2. distance between frequencies: $\left|f_{i}-f_{j}\right|=\varepsilon_{i j}$ or $\left|f_{i}-f_{j}\right| \neq \varepsilon_{i j}$;

3. polarization equality or not across paths: $p_{i}=p_{j}$ or $p_{i} \neq p_{j}$;

4. minimal distance between frequencies: $\left|f_{i}-f_{j}\right| \geq\left\{\begin{array}{lll}\gamma_{i j} & \text { if } \quad p_{i}=p_{j} \\ \delta_{i j} & \text { if } \quad p_{i} \neq p_{j} .\end{array}\right.$

In the constraints (4), the required distance between frequencies depends on their polarizations: the distance is obviously smaller if the polarizations are different $\left(\gamma_{i j} \geq \delta_{i j}\right)$

A feasible solution is an allocation for each path that satisfies the full set of constraints. Unfortunately, most problems do not have any such feasible solution, because the domains are too restrictive or the requirements are too numerous. The operator must therefore search for a "good quality" solution in terms of interference, rather than a feasible one. For this purpose, two constraint classes are introduced: 
- CI: strong or imperative constraints (1, 2, and 3 above);

- CEM: constraints of type 4 where progressive relaxation is authorized and controlled by 11 relaxation levels. Level 0 corresponds to no relaxation. Increasing from level $k$ to $k+1$ involves relaxation of some or all the frequency distances defining each constraint, the maximum level being 10:

$$
\left|f_{i}-f_{j}\right| \geq\left\{\begin{array}{l}
\gamma_{i j}^{0} \geq \gamma_{i j}^{1} \geq \ldots \geq \gamma_{i j}^{k} \geq \ldots \geq \gamma_{i j}^{10} \text { if } p_{i}=p_{j} \\
\delta_{i j}^{0} \geq \delta_{i j}^{1} \geq \ldots \geq \delta_{i j}^{k} \geq \ldots \geq \delta_{i j}^{10} \text { if } p_{i} \neq p_{j} .
\end{array}\right.
$$

In this context, a feasible solution at level $k$ is an allocation of each path satisfying all the strong constraints CI and all the CEM constraints at level $k$, noted $\mathrm{CEM}_{k}$. Such a problem is said to be $k$-feasible. Assuming that a good quality solution minimizes interference, and that the smaller $k$ is the fewer CEM constraints are relaxed, the hierarchical objective function is dependent on $k$ : first minimizing $k$, secondly minimizing the number of unsatisfied $\mathrm{CEM}_{k-1}$ constraints, and finally minimizing the number of unsatisfied CEM constraints at the levels below $k-1$.

\subsection{Formal DEFINITION}

From this physical description, modelling the FAPP as a Maximal Constraint Satisfaction Problem (Max-CSP), consists in defining the constraint network $\langle\mathcal{X}, \mathcal{D}, \mathcal{C}\rangle$, and the formal criterion to minimize $f$ :

- associating a variable $x_{i}$ to each path: $\mathcal{X}=\left\{p_{i}, i=1, \ldots, n\right\}$;

- associating to $x_{i}$ a domain $D_{i}=F_{i} \times P_{i}$, where $F_{i}$ is the set of the allowed frequencies for the path $x_{i}$ and $P_{i}$ is one of the following sets $\{-1\},\{1\}$, or $\{-1,1\}, \mathcal{D}=\bigcup_{i=1, \ldots, n} D_{i}$

- adding several imperative constraints CI between two paths $x_{i}$ and $x_{j}$ : $f_{i}=f_{j}$ or $f_{i} \neq f_{j},\left|f_{i}-f_{j}\right|=\varepsilon_{i j}$ or $\left|f_{i}-f_{j}\right| \neq \varepsilon_{i j}, p_{i}=p_{j}$ or $p_{i} \neq p_{j}$;

- adding CEM constraints where progressive relaxation is authorized:

$$
\left|f_{i}-f_{j}\right| \geq\left\{\begin{array}{l}
\gamma_{i j}^{0} \geq \cdots \geq \gamma_{i j}^{k} \quad \text { if } p_{i}=p_{j} \\
\delta_{i j}^{0} \geq \cdots \geq \delta_{i j}^{k} \quad \text { if } p_{i} \neq p_{j}
\end{array}\right.
$$

where the index $k$, increasing from 0 to 10 , indicates the relaxation level of the CEM constraints.

$\mathcal{C}=\mathrm{CI} \cup \mathrm{CEM}$;

- let $V_{(k)}$ be the set of unsatisfied $\mathrm{CEM}_{k}$ constraints. The hierarchical objective function to be minimized is:

$$
f=\left(k, V_{(k-1)}, \sum_{i<k-1} V_{(i)}\right)
$$

where $k$ is such that the problem is $k$-feasible. The formal criterion $f$ is directly related to the hierarchical optimization problem. We must therefore successively minimize $k, V_{(k-1)}$ then $\Sigma_{i<k-1} V_{(i)}$. 
Where necessary, for simplicity, we also define:

- $C_{k}=\mathrm{CI} \cup \mathrm{CEM}_{k}$ the whole constraint set at level $k$;

- $\mathrm{CI}(i, j) \subset \mathrm{CI}$ the imperative constraints involving the paths $i$ and $j$;

- $\mathrm{CEM}_{k}(i, j) \subset \mathrm{CEM}_{k}$ the relaxed constraints involving the paths $i$ and $j$.

\section{General approach For SOlving the FAPP}

Among all of the dedicated algorithms to solve frequency assignment problems $[1,4,11]$, only heuristic based approaches can produce good quality solutions in reasonable computing time for realistically sized instances. The described algorithm here is also a meta-heuristic one. Although it may seem unworkable to attempt to use a pre-processing filtering operation on an optimisation problem, our Tabu Search procedure is reinforced by a consistency technique that reduces the search space and increases the overall efficiency of the neighborhood exploration. Indeed:

- let $n$ be the path number of a FAPP instance;

- let $S$ be the non constrained search space: $S$ includes all the configuration vectors $s=\left(\left(f_{1}, p_{1}\right), \ldots,\left(f_{n}, p_{n}\right)\right)$ such as $f_{i} \in F_{i}$ and $p_{i} \in P_{i}$.

The initial size of $S$, equal to $\prod_{1}^{n}\left|F_{i}\right| \times\left|P_{i}\right|$, is huge, even for the smallest FAPP instances $\left(200 \leq n \leq 3000\right.$ and $\left.19 \leq\left|F_{i}\right| \leq 500\right)$. To cope with this difficulty, we restrict the $F_{i}$ domains by a filtering process.

The two expected advantages of such a reduction in domain size are: firstly to decrease the time complexity of the neighborhood evaluation, and secondly to avoid exploring wrong areas in the search space. That is why we hybridize our Tabu Search algorithm with an Arc-Consistent (AC) filtering process.

Another important feature of this approach, with the aim of reducing computing complexity, is that only the first component $k$ of the objective function $f$ is considered within the move heuristic. Consequently, the optimization process is transformed into a sequence of decision problems verifying the existence of a $k$ feasible configuration, where $k$ decreases from 11 to $k^{*}$ (best $k$ found). The search for a lower value of $k$ is started only if a $(k+1)$-feasible configuration has been found. Hence, we solve several CSPs rather than one Max-CSP.

To summarize, if $\mathrm{AC}$ is the filtering function of the frequency domains, and TABU the exploring function of the search space using the meta-heuristic Tabu Search, we have the general pattern described in Algorithm 1.

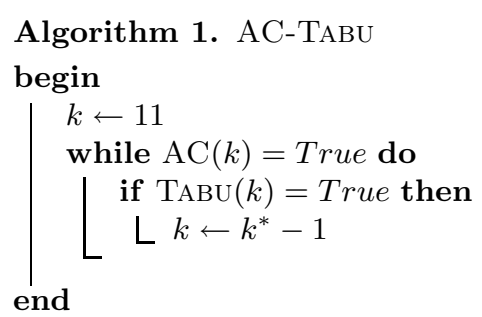


This is a very straightforward iterative procedure. When either $\mathrm{AC}(k)$ or $\operatorname{TABU}(k)$ fails then $A C$-Tabu returns $k^{*}=k+1$ as the best $k^{*}$-feasible solution found. If $\mathrm{AC}(k-1)$ fails and $\operatorname{TABU}(k)$ finds a solution, then $k$ is the optimal value. Indeed, AC provides the Lower Bound of $k$, and TABU the Upper one.

On the other hand, if $\operatorname{TABU}(k)$ finds a feasible solution, that solution may be $k^{*}$-feasible, with $k^{*}<k$. This means that TABU can jump more than one k-level.

\subsection{Filtering With AC}

Arc-consistency is a widely studied topic in constraint programming $[2,3,12,13]$. $\mathrm{AC}$ eliminates variable values with no support, since such values cannot lead to a feasible solution.

As FAPP constraints are binary, it is easy to check for every path $i(1 \leq i \leq n)$ and for every value couple $\left(f_{i}, p_{i}\right)$, if for each neighbor $j$ of $i$, there is a couple $\left(f_{j}, p_{j}\right)$ such that $\mathrm{CI}(i, j)$ and $\mathrm{CEM}_{k}(i, j)$ are satisfied. This elimination process, eliminate $\left(F_{i}, P_{i}, k\right)$, is repeated until there is no change in the domain of any variable: in this case eliminate returns False, otherwise it returns True. For more details about such algorithms, please refer to $[2,3,12]$. The main features of the following $\mathrm{AC}(k)$ procedure are:

- managing a list of updated variable domains, in order to check, at the next iteration, only the variables for which their domains have changed;

- for each $f_{i}$ and $p_{i}$ values, and for each constraint $C(i, j)$, the eliminate $\left(F_{i}, P_{i}, k\right)$ function checks another value in the domain $D_{i}$ as soon as it has found a pair $f_{j} \in D_{j}$ and $p_{j} \in P_{j}$ satisfying $C(i, j)$.

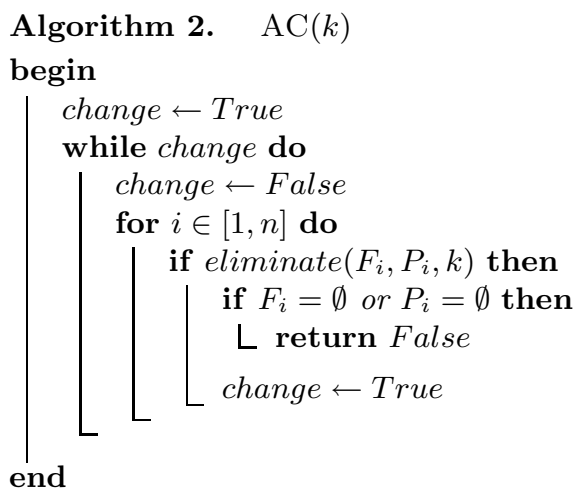

\subsection{Exploring With TABU}

Contrary to the random local search, where randomness is extensively used, the meta-heuristic Tabu Search is based on the belief that an intelligent search should include more systematic forms of guidance based on adaptive memory and learning. 
Designing a good Tabu Search requires that its main characteristics should be well defined: the effective search space and therefore the definition of the visited configurations, the neighborhood structure, the move heuristic, and the tabu list management.

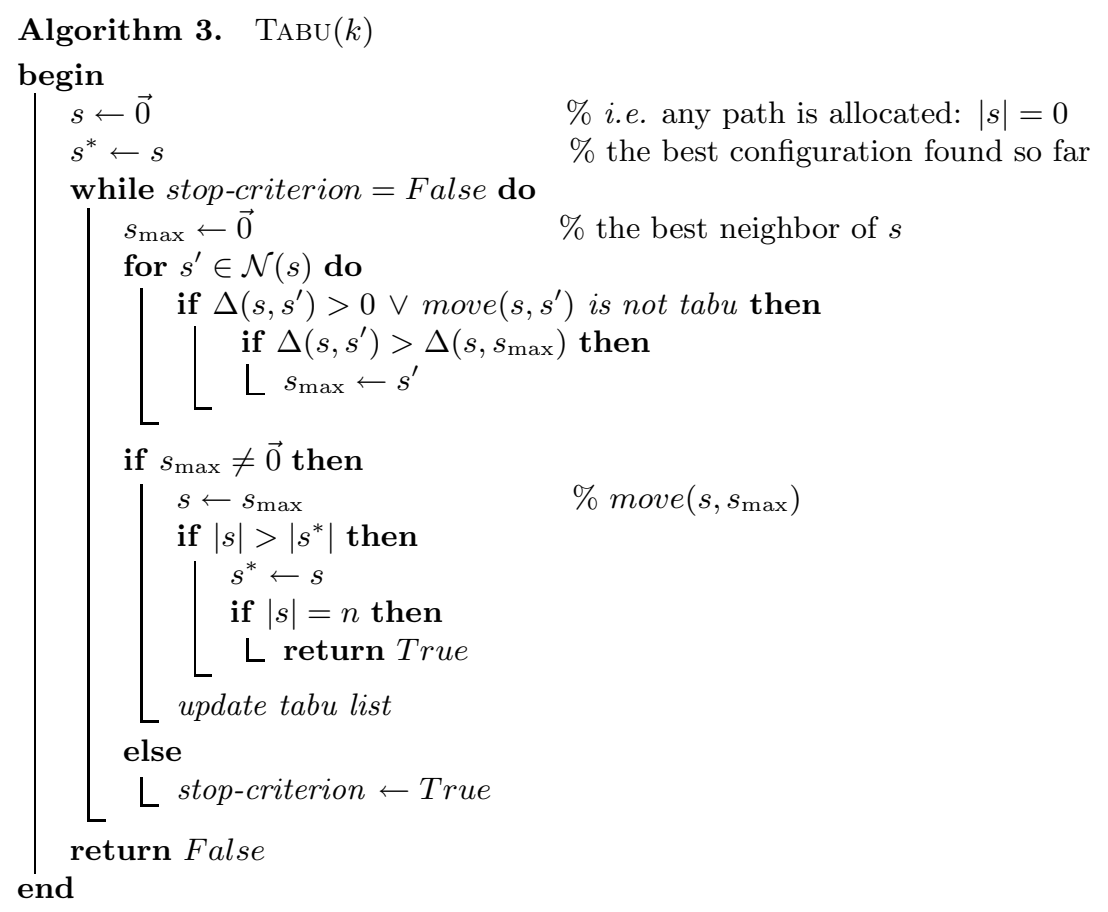

In our approach, the search space is defined over partial configurations expressed by $s=\left(v_{1}, v_{2}, \ldots, v_{n_{i}}\right)$, where $v_{i}=\left(f_{i}, p_{i}\right)$ and $n_{i}$ variables are instantiated (with $\left.n_{i}=|s|\right)$. To conform with the standard representation by $n$-ary vectors, we add a new value $u$ (for uninstantiated) to each domain $D_{i}$, for indicating that $x_{i}$ is free. Accordingly, the evaluation criterion of a configuration is the number of instantiated variables in $s$.

This improvement enabled us to work on a consistent neighborhood $\mathcal{N}(s)$. At each level $k$, the visited configurations in $\mathcal{N}(s)$ respect the CI and the $\mathrm{CEM}_{k}$ constraints. In order to build this original neighborhood, a move $\left(s, s^{\prime}\right)$, replacing the current configuration $s$ by $s^{\prime} \in \mathcal{N}(s)$, is achieved in two steps: an instantiation of a free variable (i.e. set to $u$ ), followed by consistent reparations, which are simply deinstantiations of the conflicting variables with respect to the $C_{k}$ constraints. Consequently, the selected move must improve the configuration (i.e. increase $|s|)$. Hence, the evaluation heuristic of moves from $s$ to $s^{\prime} \in \mathcal{N}(s)$, is simply $\Delta\left(s, s^{\prime}\right)=\left|s^{\prime}\right|-|s|$. Note that the $\Delta$ evaluation is carried out very quickly using efficient incremental techniques, which are now well known $[5,6,15,16]$. 
The tabu list is needed to prevent cycling, which notably occurs when we attempt to instantiate the last free variables. To avoid undoing the recent instantiation $\left(x_{i}, v_{i}\right)$, we penalize all the conflicting pairs $\left(x_{j}, v_{j}\right)$ where $x_{j}$ are the neighbors of $x_{i}$ in the constraint network, regarding the $\mathrm{CI}(i, j)$ and the $\operatorname{CEM}_{k}(i, j)$. In this way, we maintain a table counting the number of times a resource $v_{l}$ is assigned to a path $x_{i}($ freq $[i][l]++)$. So, the tabu tenure is a dynamic function based on the flip frequencies: tabu $[i][l]=$ freq $[i][l]+$ iter, where iter is the current iteration number.

The stop-criterion is either if a solution is found, or if computing time has elapsed.

With the specifications, we thus present the general TABU algorithm in 3 .

\subsection{DivERSIFICATION}

The aim of the diversification phase is to enable TABU to escape from attractive zones of the search space. For this purpose, we introduce penalties in the move heuristic. More precisely, each time a configuration $s$ such as $\forall s^{\prime} \in N(s),\left|s^{\prime}\right| \leq|s|$ is reached, we add a penalty to all the allocated paths having an unallocated neighbor in the constraint graph. This penalty value is then included in the move heuristic during the diversification phase. Hence, the line [1] in Algorithm 3 is replaced by the following code, where $\operatorname{nogood}_{\text {min }}$ is the threshold value, and penalty is the effective penalty value of the move.

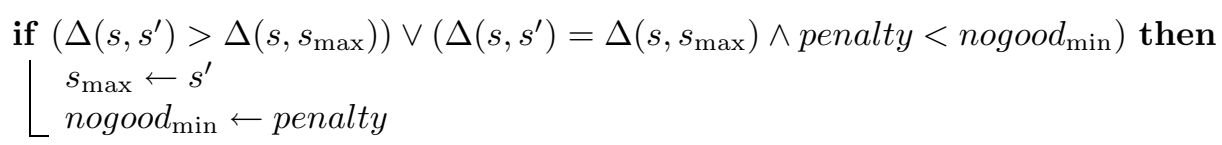

\section{NumeriCAL EXPERIMENTATION}

The algorithms were coded in $\mathrm{C}$ programming language. The running tests were carried out on an NT PC station with a Pentium III $600 \mathrm{MHz}$ cpu. The results are presented in four parts. The first describes the reduction of the search space by the filtering step. The second describes the best qualitative results obtained by the hybrid approach $A C$-Tabu. The next section then compares the results produced by our TABU and the hybrid $A C$-Tabu algorithm. This section ends with a comparison between several methods proposed for the ROADEF-2001 challenge. More information about this competition is available on the web site http://www.prism.uvsq.fr/ vdc/ROADEF/CHALLENGES/2001/

\subsection{Cutting Frequency Domains}

This first table shows some characteristics of the fapp instances, their size is given in the second part of their name: up to $\mathbf{3 0 0 0}$ variables (paths). These 
TABLE 1. Search Space reduction.

\begin{tabular}{|c|c|c|c|c|c|c|c|c|c|c|c|}
\hline fapp & $I D S$ & $F D S$ & $\Delta \%$ & $k_{0}$ & sec. & fapp & $I D S$ & $F D S$ & $\Delta \%$ & $k_{0}$ & sec. \\
\hline 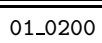 & 26963 & 12712 & $\overline{52.85}$ & 2 & 0 & 16_0260 & 47293 & 446622 & $\overline{1.42}$ & 10 & 0 \\
\hline $02 \_0250$ & 36618 & 14759 & 59.69 & 1 & 2 & 17_0300 & 64034 & 918 & 98.57 & 3 & 0 \\
\hline $03 \_0300$ & 53536 & 28212 & 47.30 & 6 & 1 & 18_0350 & 73016 & 1089 & 98.51 & 7 & 0 \\
\hline $04 \_0300$ & 61762 & 21962 & 64.44 & 0 & 4 & 19_0350 & 201074 & 3414 & 98.30 & 5 & 6 \\
\hline $05 \_0350$ & 79311 & 54177 & 31.69 & 7 & 2 & 20_0420 & 87077 & 1886 & 97.83 & 9 & 0 \\
\hline $06 \_0500$ & 108024 & 53034 & 50.91 & 4 & 6 & $21 \_0500$ & 113594 & 7745 & 93.18 & 3 & 1 \\
\hline $07 \_0600$ & 109658 & 69952 & 36.21 & 8 & 2 & $22 \_1750$ & 813037 & 10656 & 98.69 & 6 & 25 \\
\hline $08 \_0700$ & 134020 & 81933 & 38.87 & 4 & 5 & 23_1800 & 455735 & 3265 & 99.28 & 8 & 9 \\
\hline $09 \_0800$ & 121824 & 52948 & 56.54 & 2 & 6 & $24 \_2000$ & 567396 & 8328 & 98.53 & 6 & 4 \\
\hline 10_0900 & 197665 & 122050 & 38.25 & 5 & 8 & $25 \_2230$ & 610084 & 18867 & 96.91 & 2 & 6 \\
\hline $11 \_1000$ & 294634 & 152727 & 48.16 & 7 & 15 & $26 \_2300$ & 635123 & 14217 & 97.76 & 6 & 4 \\
\hline $12 \_1500$ & 436967 & 164613 & 62.33 & 1 & 70 & $27 \_2550$ & 588188 & 93768 & 84.06 & 4 & 8 \\
\hline $13 \_2000$ & 320494 & 144873 & 54.80 & 2 & 21 & $28 \_2800$ & 2087947 & 63597 & 96.95 & 2 & 66 \\
\hline $14 \_2500$ & 774322 & 320458 & 58.61 & 3 & 92 & 29_2900 & 1477634 & 6435 & 99.56 & 5 & 23 \\
\hline $15 \_3000$ & 515606 & 306127 & 40.63 & 4 & 24 & 30_3000 & 1942250 & 80703 & 95.84 & 6 & 103 \\
\hline
\end{tabular}

problems contain up to $\mathbf{2} \mathbf{0 8 7 9 4 7}$ total domain values, and up to $\mathbf{6 7 8 9 8}$ binary constraints.

Table 1 gives the Initial Domain Size $\left(I D S=\sum_{1}^{n}\left|D_{i}^{0}\right|\right)$ and the Filtered one $\left(F D S=\sum_{1}^{n}\left|D_{i}^{f}\right|\right)$. Columns $k_{0}$ indicate the highest unfeasible level encountered by the $A C$-Tabu procedure, and columns sec. give the computing time in seconds for the whole iterative filtering process. This table shows that no more than two minutes are required by $A C$-Tabu to reduce the domains of the largest instances of this benchmark.

Apart from the fapp16_0260 problem, these benchmarks can be divided into two subsets. The first one (from 01 to 15) contains the instances where the domain size is reduced by nearly half. In the second one (from 17 to 30), domains are reduced more than $90 \%$.

\subsection{HYBRID APPROACH RESULTS}

This part begins with the results obtained by the hybrid approach after 1 computing hour. Only one run (with the 0 random seed) was carried out in this experiment.

For each instance, the Table 2 specifies:

$-k$, the lowest level where a solution is found. This value is underlined when $A C$-Tabu proves its optimality;

- $V_{k-1}$, the number of unsatisfied CEM constraints at level $k-1$;

- $\sum V_{k-2}$, the sum of the unsatisfied CEM constraints under the $k-1$ level;

- $t_{1}$, the elapsed time in seconds required to reach the best value of $k$;

- and $t_{2}$, the elapsed time required to obtain the best configuration (considering the three components of the objective function). 
TABLE 2. AC-Tabu results after 1 computing hour.

\begin{tabular}{|r|r|r|r|r|r||r|r|r|r|r|r|}
\hline fapp & $k$ & $V_{k-1}$ & $\sum V_{k-2}$ & \multicolumn{1}{|c|}{$t_{1}$} & \multicolumn{1}{c|}{$t_{2}$} & fapp & $k$ & $V_{k-1}$ & $\sum V_{k-2}$ & $t_{1}$ & $t_{2}$ \\
\hline \hline 01_0200 & 4 & 4 & 203 & 2 & 1839 & $16 \_0260$ & $\underline{11}$ & 382 & 3864 & 0 & 2976 \\
\hline 02_0250 & $\underline{2}$ & 16 & 173 & 19 & 19 & $17 \_0300$ & $\underline{4}$ & 4 & 36 & 1 & 1 \\
\hline 03_0300 & $\underline{7}$ & 28 & 835 & 30 & 34 & $18 \_0350$ & $\underline{8}$ & 4 & 55 & 1 & 1 \\
\hline 04_0300 & $\underline{1}$ & 97 & 0 & 26 & 2595 & $19 \_0350$ & $\underline{6}$ & 3 & 66 & 16 & 16 \\
\hline 05_0350 & 11 & 1 & 1836 & 1 & 3571 & 20_0420 & $\underline{10}$ & 6 & 133 & 2 & 2 \\
\hline 06_0500 & $\underline{5}$ & 30 & 764 & 232 & 233 & 21_0500 & $\underline{4}$ & 2 & 12 & 2 & 2 \\
\hline 07_0600 & $\underline{9}$ & 85 & 3039 & 107 & 433 & 22_1750 & $\underline{7}$ & 22 & 383 & 76 & 76 \\
\hline 08_0700 & $\underline{5}$ & 67 & 1131 & 21 & 35 & 23_1800 & $\underline{9}$ & 16 & 189 & 31 & 31 \\
\hline 09_0800 & $\underline{3}$ & 71 & 707 & 1789 & 1796 & 24_2000 & $\underline{7}$ & 9 & 91 & 17 & 17 \\
\hline 10_0900 & $\underline{6}$ & 74 & 1877 & 174 & 372 & 25_2230 & $\underline{3}$ & 7 & 33 & 19 & 19 \\
\hline 11_1000 & $\underline{8}$ & 105 & 4278 & 63 & 139 & 26_2300 & $\underline{7}$ & 8 & 75 & 18 & 18 \\
\hline 12_1500 & 4 & 87 & 2138 & 2873 & 2873 & 27_2550 & $\underline{5}$ & 9 & 46 & 21 & 21 \\
\hline 13_2000 & 5 & 126 & 3469 & 1020 & 1020 & 28_2800 & $\underline{3}$ & 38 & 125 & 165 & 203 \\
\hline 14_2500 & 6 & 211 & 6302 & 1875 & 2950 & 29_2900 & $\underline{6}$ & 25 & 280 & 86 & 86 \\
\hline 15_3000 & $\underline{5}$ & 198 & 4770 & 2534 & 2807 & 30_3000 & $\underline{7}$ & 36 & 798 & 268 & 275 \\
\hline
\end{tabular}

TABLE 3. Improved results after 3 hours of computing.

\begin{tabular}{|c|r|r|r|r|r|}
\hline fapp & \multicolumn{1}{c|}{$k$} & $V_{k-1}$ & $\sum V_{k-2}$ & \multicolumn{1}{c|}{$t_{1}$} & \multicolumn{1}{c|}{$t_{2}$} \\
\hline \hline 01_0200 & 4 & 4 & 139 & 2 & 10194 \\
\hline 05_0350 & 11 & 1 & 1683 & 1 & 5446 \\
\hline 12_1500 & $\underline{2}$ & 57 & 1263 & 5384 & 5388 \\
\hline 13_2000 & $\underline{3}$ & 165 & 2253 & 7345 & 7686 \\
\hline 14_2500 & 5 & 168 & 4700 & 10450 & 10450 \\
\hline 16_0260 & $\underline{11}$ & 358 & 3656 & 0 & 8543 \\
\hline
\end{tabular}

Regarding the feasibility level, most of the $k$ are proved optimal (25 of the 30 instances). Obviously, the instances for which no $k^{*}$ is found are those belonging to the first subset described above. Now, we shall see what happens if we give more cpu time to the hybrid algorithm process.

Table 3 shows only the improved solutions reached after 3 computing hours. Unfortunately, no improvement has been obtained for the majority of the instances. Nevertheless, we can see again that it only concerns problems belonging to the first subset of fapp instances. Elsewhere, two new optimal values of $k$ are proved (for the 12_1500 and the 13_2000 problems).

\subsection{TABU versus AC-TABU}

This section compares the behaviors of TABU alone and the hybrid $A C$-Tabu algorithm. These algorithms were executed 8 times with seed values varying from 0 to 7 , and one hour of computing time.

Table 4 focuses on the $k$-feasibility level and gives the best $\left(k_{\min }\right)$ and the worst $\left(k_{\max }\right)$ solutions found by the TABU and hybrid $A C$-Tabu processes. 
TABLE 4. Best and worst results for the level of feasibility.

\begin{tabular}{|c|c|c|c|c|c|c|c|c|c|}
\hline \multirow[b]{2}{*}{ fapp } & \multicolumn{2}{|c|}{$A C-T a b u$} & \multicolumn{2}{|c|}{ TABU } & \multirow[b]{2}{*}{ fapp } & \multicolumn{2}{|c|}{$A C-T a b u$} & \multicolumn{2}{|c|}{ TABU } \\
\hline & $k_{\min }$ & $k_{\max }$ & $k_{\min }$ & $k_{\max }$ & & $k_{\min }$ & $k_{\max }$ & $k_{\min }$ & $k_{\max }$ \\
\hline $01 \_0200$ & 4 & 4 & 4 & 4 & $16 \_0260$ & 11 & 11 & 11 & 11 \\
\hline $02 \_0250$ & 2 & 2 & 2 & 3 & 17_0300 & 4 & 4 & 4 & 4 \\
\hline 03_0300 & 7 & 7 & 7 & 7 & 18_0350 & 8 & 8 & 8 & 8 \\
\hline $04 \_0300$ & 1 & 1 & 1 & 11 & 19_0350 & 6 & 6 & 6 & 9 \\
\hline $05 \_0350$ & 11 & 11 & 11 & 11 & $20 \_0420$ & 10 & 10 & 10 & 10 \\
\hline $06 \_0500$ & 5 & 5 & 5 & 6 & 21_0500 & 4 & 4 & 4 & 5 \\
\hline $07 \_0600$ & 9 & 10 & 9 & 10 & $22 \_1750$ & 7 & 7 & 7 & 11 \\
\hline 08_0700 & 5 & 6 & 5 & 6 & 23_1800 & 9 & 9 & 9 & 11 \\
\hline 09_0800 & 3 & 4 & 3 & 8 & 24_2000 & 7 & 7 & 7 & 11 \\
\hline 10_0900 & 6 & 7 & 6 & 11 & 25_2230 & 3 & 3 & 10 & 11 \\
\hline $11 \_1000$ & 8 & 9 & 8 & 11 & 26_2300 & 7 & 7 & 7 & 10 \\
\hline $12 \_1500$ & 4 & 7 & 6 & 8 & 27_2550 & 5 & 5 & 11 & 11 \\
\hline 13_2000 & 5 & 6 & 6 & 6 & 28_2800 & 3 & 3 & 9 & 11 \\
\hline $14 \_2500$ & 6 & 6 & 7 & 11 & 29_2900 & 6 & 6 & 8 & 12 \\
\hline 15_3000 & 5 & 6 & 7 & 11 & 30_3000 & 7 & 7 & 11 & 11 \\
\hline
\end{tabular}

The gap between $k_{\text {min }}$ and $k_{\max }$ is higher for TABU, than for $A C$-Tabu, notably on the second subset of the benchmark. In conclusion, the behavior of $A C$-Tabu is more stable than that of TABU alone.

Note that the results of fapp12_1500, fapp13_2000 and fapp14_2500 do not contradict those from Table 3: it simply means that $A C$-Tabu finds a better solution in 3 hours with 0 random seed than in $8 \times 1$ hour with different random seeds.

\subsection{Comparison With others methods}

We will now present the results obtained during the ROADEF-2001 challenge, which took place in FRANCORO III, in the city of Québec (Canada).

The three compared algorithms (apart from ours) were developed by Caseau $(\mathrm{MH}+\mathrm{PPC})$, the Bisaillon team (Tabu), and the Michelon junior team $(\mathrm{LNS}+\mathrm{PPC})$.

The (MH+PPC) algorithm combines some constraint propagation with metaheuristics. More precisely, the algorithm increases the level, and at each one it shaves by strong consistency, shuffles by a Large Neighborhood Search (LNS) [14], which gives a non-feasible solution, and finally tries to obtain a feasible solution by a Limited Discrepancy Search (LDS) [10], such as a Variable Neighborhood local search (VNS) [9]. 
The second approach (Tabu) [7] is a local search approach based on Tabu Search that includes some original features, including a specialized neighborhood, heuristics to determine critical variables and values, different diversification techniques, an auto-adaptative mechanism to set the tabu list and finally, a pre-processing operation based on consistency techniques inherited from constraint programming. It is used to treat three different problems:

- Constraint Satisfaction Problem to find a solution at level $k$;

- the problem which satisfies all the constraints at level $k+1$, and as much as possible at level $k$;

- the problem which satisfies all the constraints at level $k+1$, and minimizes the objective function.

The Michelon junior team solves the FAPP by using a meta-heuristic based on a Large Neighborhood Search [14] and Constraint Propagation. Also, they relax certain constraints to obtain a Maximal Cover Tree. Indeed, their algorithm works in three phases: first it computes a lower bound of $k$, then it searches for a solution at this level $k$, and finally it improves this solution with respect to the two last points of the objective function. So as to compare several methods, in terms of quality, we have just given the three values of the objective function: $k$ (underlined when the method proves optimality), the unsatisfied constraint number at level $k-1$, and the sum of the unsatisfied constraints at the levels below $k-1$.

The $A C$-Tabu column gives the results obtained by our method during the challenge. Note that they have been improved since the competition. Indeed, we have polished the code, and experiments are made on different computers. More details about the challenge, benchmarks and all the results can be found on the web site http://www.prism.uvsq.fr/ vdc/ROADEF/CHALLENGES/2001/

The main test condition is a computing time limited to one hour on a Pentium III, 500 Mhz, 128 Mo.

Regarding only the level $k, \mathrm{MH}+\mathrm{PPC}$ found the optimal $k 26$ times, Tabu 27 times, LNS+PPC 27 times, and $A C$-Tabu 28 times. It seems that there is a problem with the LNS $+\mathrm{PPC}$ method, because in 4 instances, it could not find a solution $(k=12)$ and in several others it found no solution better than 11 .

Regarding the two other components of the objective function, Tabu without $\mathrm{AC}$ gave the best results 27 times, although it could not prove optimality. In fact, the time spent on the PPC techniques in the other methods, was spent to improve the full objective function.

This method comparison reveals that the most difficult instances of the benchmark were 12_1500,13_2000 and 14_2500.

To conclude, Table 5 highlights the effectiveness of approximate methods combined with some arc-consistent processes on very large instances. 
TABLE 5. Comparison with three other methods.

\begin{tabular}{|c|c|c|c|c|c|c|c|c|c|c|c|c|}
\hline & \multicolumn{3}{|c|}{$\mathrm{MH}+\mathrm{PPC}$} & \multicolumn{3}{|c|}{ Tabu } & \multicolumn{3}{|c|}{$\mathrm{LNS}+\mathrm{PPC}$} & \multicolumn{3}{|c|}{$A C-T a b u$} \\
\hline fapp & $k$ & $V_{k-1}$ & $\sum V_{k-2}$ & $k$ & $V_{k-1}$ & $\sum V_{k-2}$ & $k$ & $V_{k-1}$ & $\sum V_{k-2}$ & $k$ & $V_{k-1}$ & $\sum V_{k-2}$ \\
\hline 01_0200 & $\overline{\underline{4}}$ & $\overline{6}$ & $\overline{279}$ & $\overline{4}$ & $\overline{4}$ & $\overline{\overline{56}}$ & $\overline{\underline{4}}$ & 5 & $\overline{210}$ & $\overline{4}$ & $\overline{14}$ & $\overline{233}$ \\
\hline 02_0250 & $\underline{2}$ & 18 & 248 & 2 & 7 & 86 & $1 \overline{1}$ & 1 & 435 & $\underline{2}$ & 20 & 195 \\
\hline 03_0300 & $\overline{7}$ & 27 & 1076 & 7 & 10 & 341 & 11 & 1 & 1211 & $\overline{7}$ & 32 & 892 \\
\hline 04_0300 & $\underline{1}$ & 164 & 0 & 1 & 31 & 0 & 2 & 1 & 282 & $\overline{1}$ & 184 & 0 \\
\hline 05_0350 & $1 \overline{1}$ & 892 & 12364 & 11 & 1 & 372 & 11 & 97 & 3459 & $1 \overline{1}$ & 364 & 5694 \\
\hline 06_0500 & $\underline{5}$ & 53 & 1029 & 5 & 12 & 246 & 6 & 1 & 1086 & $\underline{5}$ & 31 & 811 \\
\hline 07_0600 & $\underline{\overline{9}}$ & 132 & 4419 & 9 & 22 & 714 & 12 & - & - & $\underline{\overline{9}}$ & 106 & 3375 \\
\hline 08_0700 & $\underline{5}$ & 53 & 1359 & 5 & 16 & 266 & 11 & 3 & 2144 & $\underline{5}$ & 73 & 1225 \\
\hline 09_0800 & $\underline{3}$ & 63 & 937 & 3 & 28 & 195 & 4 & 2 & 999 & $\underline{3}$ & 104 & 846 \\
\hline 10_0900 & $\underline{6}$ & 82 & 2365 & 6 & 18 & 475 & 11 & 4 & 3661 & $\underline{6}$ & 103 & 2003 \\
\hline 11_1000 & $\underline{8}$ & 119 & 5206 & 8 & 8 & 1015 & 11 & 8 & 6146 & $\underline{8}$ & 119 & 4191 \\
\hline 12_1500 & $\overline{7}$ & 0 & 6538 & 3 & 83 & 1698 & 11 & 647 & 13797 & $\underline{2}$ & 62 & 1310 \\
\hline 13_2000 & 7 & 229 & 7503 & 3 & 49 & 2003 & 11 & 671 & 15145 & $\overline{5}$ & 132 & 3645 \\
\hline 14_2500 & 8 & 18 & 10661 & 4 & 35 & 3485 & 11 & 1209 & 24751 & 5 & 217 & 5045 \\
\hline 15_3000 & 7 & 333 & 9988 & 5 & 15 & 1569 & 11 & 1060 & 22898 & $\underline{5}$ & 192 & 4727 \\
\hline $16 \_0260$ & $\underline{11}$ & 572 & 5779 & 11 & 5 & 56 & $\underline{11}$ & 590 & 5968 & $\underline{11}$ & 514 & 5189 \\
\hline 17_0300 & $\underline{4}$ & 4 & 36 & 4 & 4 & 34 & $\underline{4}$ & 4 & 36 & $\underline{4}$ & 4 & 36 \\
\hline 18_0350 & 8 & 4 & 55 & 8 & 4 & 55 & $\underline{8}$ & 4 & 57 & $\underline{8}$ & 4 & 59 \\
\hline 19_0350 & 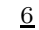 & 3 & 79 & 6 & 2 & 51 & $\underline{6}$ & 2 & 60 & $\underline{6}$ & 3 & 70 \\
\hline 20_0420 & $\underline{10}$ & 6 & 145 & 10 & 5 & 97 & $\underline{10}$ & 5 & 106 & $\underline{10}$ & 7 & 142 \\
\hline 21_0500 & $\underline{4}$ & 2 & 12 & 4 & 2 & 10 & $\underline{4}$ & 2 & 12 & $\underline{4}$ & 2 & 12 \\
\hline 22_1750 & $\underline{7}$ & 16 & 356 & 7 & 15 & 187 & $\underline{7}$ & 15 & 292 & $\underline{7}$ & 25 & 503 \\
\hline 23_1800 & $\underline{9}$ & 17 & 197 & 9 & 16 & 187 & 12 & - & - & $\underline{9}$ & 17 & 197 \\
\hline 24_2000 & $\underline{7}$ & 7 & 90 & 7 & 6 & 71 & $\underline{7}$ & 6 & 77 & $\underline{7}$ & 9 & 91 \\
\hline 25_2230 & $\underline{3}$ & 7 & 33 & 3 & 7 & 32 & $\underline{3}$ & 7 & 34 & $\underline{3}$ & 7 & 33 \\
\hline $26 \_2300$ & 7 & 10 & 81 & 7 & 9 & 74 & 7 & 9 & 75 & 7 & 10 & 86 \\
\hline 27_2550 & $\underline{5}$ & 7 & 46 & 11 & 4 & 64 & $\underline{5}$ & 4 & 22 & $\underline{5}$ & 11 & 54 \\
\hline 28_2800 & $\underline{3}$ & 32 & 129 & 3 & 13 & 32 & $\underline{3}$ & 14 & 72 & $\underline{3}$ & 42 & 142 \\
\hline 29_2900 & $\underline{6}$ & 28 & 351 & 6 & 25 & 239 & 12 & - & - & $\underline{6}$ & 25 & 310 \\
\hline 30_3000 & $\underline{7}$ & 17 & 602 & 11 & 1166 & 12029 & 12 & - & - & $\underline{7}$ & 48 & 1045 \\
\hline
\end{tabular}

\section{Conclusion}

In this paper we have presented a hybrid approach to solve the Frequency Assignment Problem with Polarization. AC-Tabu combines some arc-consistent techniques and an original Tabu Search process, with a large neighborhood exploration by means of maintaining consistency. It produces very good results on large-sized FAPP instances.

TABU alone proves its own worth. Indeed, it provides most of the best results but at the same time, some of the worst ones. There are two main reasons that justify the filtering step: the behavior regularity of the hybrid search algorithm, and the proven optimality of the $k$-feasibility level, for most of the FAPP instances.

Some improvements to this approach are still to be expected. Two perspectives for future work can be identified. Firstly, making AC faster by studying the most recent $\mathrm{AC}$ algorithms [3] and adapting to the FAPP constraint semantic. Secondly, considering the two other objective function components $V_{k-1}$ and $\sum_{i<k-1} V_{i}$ in the TABU procedure. 
Acknowledgement. Special thanks to Van-Dat Cung (PRiSM-UVSQ), Thierry Defaix (CELAR-DGA) and Maurice Diamantini (ENSTA) for allowing us to use their FAPP definition. Thanks also to Gérard Verfaillie for fruitful discussion about some arc-consistent techniques.

\section{REFERENCES}

[1] K.I. Aardal, C.A.J. Hurkens, J.K. Lenstra and S.R. Tiourine, Algorithms for Frequency Assignment Problems. CWI Quartely 9 (1996) 1-9.

[2] C. Bessière, Arc-consistency and Arc-consistency again. Artif. Intell. 65 (1994) 179-190.

[3] C. Bessière and J.C. Régin, Refining the Basic Consistency Propagation Algorithm, in the 17th International Joint Conference on Artificial Intelligence (IJCAI'01), SeattleWashington, USA (2001) 309-315.

[4] R. Dorne, Étude des méthodes heuristiques pour la coloration, la T-coloration et l'affectation de fréquences. Ph.D. thesis, Université Montpellier II Sciences et Techniques du Languedoc (mai 1998).

[5] C. Fleurent and J.A. Ferland, Genetic and Hybrid Algorithms for Graph Coloring. Ann. Oper. Res. 63 (1996) 437-461.

[6] P. Galinier, Étude des métaheuristiques pour la résolution du problème de satisfaction de contraintes et de coloration de graphes. Ph.D. thesis, Université Montpellier II Sciences et Techniques du Languedoc (janvier 1999).

[7] P. Galinier, S. Bisaillon, M. Gendreau and P. Soriano, Solving the Frequency Assignment Problem with Polarization by Local Search and Tabu, in The 6th Triennal Conference of the International Federation of Operational Research Societies (IFORS'O2), University of Edinburgh, UK (July 2002) 8-12.

[8] F. Glover and M. Laguna, Tabu Search. Kluwer Academic Publishers (1997).

[9] P. Hansen and N. Mladenovic, Variable Neighborhood Search. Comput. Oper. Res. 24 (1997) 1097-1100.

[10] W.D. Harvey and M.L. Ginsberg, Limited Discrepancy Search, in Proc. of the 14th International Joint Conference on Artificial intelligence (IJCAI-95) (1995) 607-613.

[11] M. Jiang, Méthodes Approchées pour le Problème de Coloriage Généralisé Application au Problème d'Allocation de Fréquences Multiservices dans l'Aviation Civile. Ph.D. thesis, Université Versailles Saint-Quentin-en-Yvelines (novembre 1996).

[12] A.K. Mackworth, Consistency in Networks of Relations. Artif. Intell. 8 (1977) 99-118.

[13] R. Mohr and T.C. Henderson, Arc and Path Consistency revisited. Artif. Intell. 28 (1986) 225-233.

[14] P Shaw, Using Constraint Programming and Local Search Methods to Solve Vehicle Routing Problems, in Principle and Pratice of Constraint Programming, CP'g8 (October 1998).

[15] M. Vasquez, Résolution en variables 0-1 de problèmes combinatoires de grande taille par la méthode tabou. Ph.D. thesis, Université d'Angers, UFR de Sciences (December 2000).

[16] M. Vasquez and J.K. Hao, A "Logic-Constrained" Knapsack Formulation and a Tabu Algorithm for the Daily Photograph Scheduling of an Earth Observation Satellite. Comput. Optim. Appl. 20 (2001) 137-157. 